University of Nebraska - Lincoln

DigitalCommons@University of Nebraska - Lincoln

July 1970

\title{
Photoionization of Argon and Xenon Including Final-State Correlation
}

Anthony F. Starace

University of Nebraska-Lincoln, astarace1@unl.edu

Follow this and additional works at: https://digitalcommons.unl.edu/physicsstarace

Part of the Physics Commons

Starace, Anthony F., "Photoionization of Argon and Xenon Including Final-State Correlation" (1970). Anthony F. Starace Publications. 1.

https://digitalcommons.unl.edu/physicsstarace/1

This Article is brought to you for free and open access by the Research Papers in Physics and Astronomy at DigitalCommons@University of Nebraska - Lincoln. It has been accepted for inclusion in Anthony F. Starace Publications by an authorized administrator of DigitalCommons@University of Nebraska - Lincoln. 
We see that there is much that atomic scattering can borrow from and contribute to high-energy nuclear physics. Clearly, because of costs, atoms and their excited states are more accessible to many experimental physicists than are nucleons and their excited states. It is hoped that this work, which explores only a few of the connections, will serve a useful function towards fur - thering the theoretical interchange between the two fields.

\section{ACKNOWLEDGMENTS}

It is a pleasure to thank Dr. A. Gersten and Dr. J. Purcell for stimulating discussions. We thank R. Berg for the use of his code to solve Schrödinger's equation.
*Address after August 31, 1970: California State College at Los Angeles, Los Angeles, Calif. 90032.

$\dagger$ This work was supported in part by the U.S. Atomic Energy Commission, under Grant No. AEC-AT-(40-1)3798.

${ }^{1}$ J. E. Purcell, R. A. Berg, and A. E. S. Green, preceding paper, Phys. Rev. A 2 , 111 (1970).

${ }^{2}$ T. Ueda and A. E. S. Green, Phys. Rev. 174, 1304 (1968); A. E. S. Green and T. Sawada, Rev. Mod. Phys. 39, 594 (1967).

${ }^{3}$ Handbook of Mathematical Functions, edited by M. Abramowitz and I. A. Stegun (Dover, New York, 1965).

${ }^{4}$ I. S. Gradshteyn and I. M. Ryzhik, Tables of Integrals, Series and Products (Academic, New York, 1965).

${ }^{5}$ T. F. O'Malley, L. Spruch, and L. Rosenberg, J. Math. Phys. 2, 491 (1961).

${ }^{6}$ L. Spruch, R. F. O'Malley, and L. Rosenberg, Phys. Rev. Letters $\underline{5}, 375$ (1960).

${ }^{7}$ R. W. LaBahn and J. Callaway, Phys. Rev. 180, 91 (1969).
${ }^{8}$ J. Callaway, R. W. LaBahn, R. T. Pu, and W. M. Duxler, Phys. Rev. 168, 12 (1968).

${ }^{9}$ S. K. Dutta, W. A. Wilson, and A. E. S. Green, J. Math. Phys. $\underline{9}, 578$ (1968).

${ }^{10}$ L. Vriens, C. E. Kuyatt, and S. R. Mielczarek, Phys. Rev. 170, 163 (1968).

${ }^{11} \mathrm{R}$. W. LaBahn (private communication).

${ }^{12}$ J. P. Bromberg, J. Chem. Phys. 50, 3906 (1969).

${ }^{13}$ A. E. S. Green, D。 L. Sellin, and A. Zachor, Phys. Rev. 184, 1 (1969).

${ }^{14}$ A. E. S. Green (unpublished).

${ }^{15}$ W. K. H. Panofsky, in Proceedings of the 14th International Conference on High-Energy Physics, Vienna, 1968, edited by J. Prentki and J. Steinberger (CERN, Geneva, 1968).

${ }^{16} \mathrm{~J}$. D. Bjorken and E. A. Paschos, Stanford Linear Accelerator Report No. SLAL-PUB-572 (unpublished).

${ }^{17}$ A. E. S. Green and S. K. Dutta, J. Geophys. Res. 72, 3933 (1967).

\title{
Photoionization of Argon and Xenon Including Final-State Correlation*
}

\author{
Anthony F. Starace ${ }^{\dagger}$ \\ Department of Physics, The University of Chicago, Chicago, Illinois 60637 \\ (Received 6 February 1970)
}

\begin{abstract}
Effects of final-state correlation on the photoionization cross sections for the $3 p$ shell in argon and the $4 d$ shell in xenon are calculated by the reaction-matrix method, starting from the Herman-Skillman model Hamiltonian, for photoelectron energies $0 \leqslant \epsilon \leqslant 1.2$ a.u. and $0 \leqslant \epsilon \leqslant 2.52$ a.u., respectively. The results for argon complement Hartree-Fock and other final-state correlation calculations; the results for xenon are the first $a b$ initio calculations of this kind. Although length and velocity forms of the transition matrix element agree in the initial local-field approximation, they are shown to diverge necessarily when only the final state is improved.
\end{abstract}

\section{INTRODUCTION}

Independent electron theory, whether based on a local central-field potential or a nonlocal (Hartree-Fock) potential, fails conspicuously to account for the photoionization cross section of the $3 p$ shell in argon and the $4 d$ shell in xenon near threshold, where electron correlation is impor- tant. ${ }^{1}$ For argon, Hartree- Fock calculations ${ }^{2,3}$ fail to reproduce the experimentally observed knee in the cross section profile, while for both argon $^{2}$ and xenon, ${ }^{4}$ calculations of the cross section using the Herman-Skillman ${ }^{5}$ (HS) local potential give shifted narrow peaks two or more times larger than experiment. Fano and Cooper ${ }^{6}$ have classified correlation effects in the continuum as 
being either intrachannel (mixing of configurations which differ only in the energy index of a single electron, i. e., $\epsilon$ in the continuum and $n$ in the discrete; the set of all such configurations being called a channel) or interchannel (mixing of configurations belonging to different channels). Calculations with a Hartree-Fock nonlocal potential include intrachannel correlation from the start, since matrix elements of the residual interaction between states of the same channel are zero. ${ }^{7}$ However, the nonlocality creates theoretical inconsistencies, ${ }^{3,8}$ discussed later on, as well as additional labor.

The present paper extends the computational evidence, particularly for xenon, starting from a local central field (HS) and calculating the intrachannel effect nonperturbatively by the reaction matrix method. ${ }^{1,9}$ Our results for argon are meant to complement both the Hartree-Fock results ${ }^{2,3}$ and more detailed calculations including both intrachannel and interchannel interaction. ${ }^{10}$ Our results for xenon are the first $a b$ initio calculations of this kind, previous theoretical work comprising plasma-type treatments ${ }^{1,11,12}$ and a HS calculation. ${ }^{4}$

\section{THEORY}

Detailed discussions are given elsewhere. ${ }^{1,9}$ Briefly, we split the spin-independent Hamiltonian into the HS model Hamiltonian

$$
H_{\text {mod }}=\sum_{i}\left(-\frac{1}{2} \nabla_{i}^{2}+V_{\mathrm{HS}}\left(r_{i}\right)\right),
$$

and a residual interaction

$$
V=-\sum_{i}\left(Z / r_{i}+V_{\mathrm{HS}}\left(r_{i}\right)\right)+\sum_{i>j} \frac{1}{r_{i j}}
$$

Atomic units are used throughout this paper. We assume the model describes the ground state far better than it does excited states, and hence are concerned only with improving final-state wave functions. In an exact treatment, the final-state eigenfunctions (for the photoionized Ar or Xe core plus ejected electron) are obtained by diagonalizing the submatrix of $H_{\text {mod }}+V$ defined by an infinitedimensional basis of ${ }^{1} P^{\circ}$ Slater determinants of $H_{\text {mod }}$ eigenfunctions. By necessity, we must use a finite basis, hence the following approximations: (i) We assume the ion core of the final state to be well described by the relevant discrete eigenfunctions of $H_{\text {mod }}$. We concern ourselves only with the improvement of the ejected electron's wave function. (ii) In the independent-particle theory, the cross section for photoionization of an electron which undergoes the angular momentum change $l \rightarrow l+1$ is an order of magnitude larger than for the change $l \rightarrow l-1$. Hence, we limit ourselves to improving the cross section for $l \rightarrow l+1$ transitions and add this to the HS cross section for $l \rightarrow l$ - 1. (iii) Since the matrix element of $V$ between two continuum wavefunctions (normalized per unit energy) is dimensionless, its magnitude compared to unity measures the strength of the interaction. We use the criterion that we need only diagonalize the matrix of $V$ in the region where its matrix elements are greater than about 0.1 , which enables us to work with a finite-sized $V$ matrix.

Our finite basis of model final states comprises then the Slater determinants $\left|1 s^{2} \ldots 3 p^{5} \epsilon d\left({ }^{1} P^{\circ}\right)\right\rangle$ for argon and $\left|1 s^{2} \ldots 4 d^{9} 5 s^{2} 5 p^{6} \epsilon f\left({ }^{1} P^{\circ}\right)\right\rangle$ for xenon. The energy index $\epsilon$ of the excited electron runs from the discrete to the continuum, taking 12 values for argon (up to $1.6 \mathrm{a}$.u. ) and 15 values for xenon (up to 3.26 a.u.), which were chosen so that matrix elements may be interpolated over the energy range considered. In what follows, we indicate members of the basis only by the energy index of the excited electron, e.g. , $|\epsilon\rangle$.

Having defined a finite submatrix of the Hamiltonian dealing with intrachannel interactions over a limited energy range, we proceed to find its eigenvectors. Each of these, denoted $\left|\Psi_{E}\right\rangle$, is a superposition of basis states defined in terms of a reaction matrix $K(E)$ :

$$
\begin{aligned}
\left|\Psi_{E}\right\rangle & =\left\{|E\rangle+P \int d \epsilon|\epsilon\rangle \frac{1}{E-\epsilon}\langle\epsilon|K(E)| E\rangle\right\} \\
& \times\left[1+\pi^{2}\langle E|K(E)| E\rangle^{2}\right]^{-1 / 2},
\end{aligned}
$$

where $P$ indicates the principal part is taken at the singularity, and where a sum over discrete states is implied. The nonsingular reaction matrix, common in the theory of collisions, satisfies the equation

$$
\begin{aligned}
\left\langle\epsilon|K(E)| \epsilon^{\prime}\right\rangle= & \left\langle\epsilon|V| \epsilon^{\prime}\right\rangle+P \int d \epsilon^{\prime \prime}\left\langle\epsilon|V| \epsilon^{\prime \prime}\right\rangle \\
& \times \frac{1}{E-\epsilon^{\prime \prime}}\left\langle\epsilon^{\prime \prime}|K(E)| \epsilon^{\prime}\right\rangle .
\end{aligned}
$$

[In this paper, $E$ and $\epsilon$ indicate energies of the excited electron only. This convention implies that those matrix elements of $V$ due to the interaction between pairs of unexcited core electrons (occurring only when $\epsilon=\epsilon^{\prime}$ ) have been subtracted from the matrix $\left\langle\epsilon|V| \epsilon^{\prime}\right\rangle$. This subtraction was not implied in Eq. (6. $\left.7^{\prime}\right)$ of Ref. 1.]

Alternative forms of the transition matrix element between HS states satisfy the equality

$$
-i\left(E-E_{g}\right)\left\langle E_{g}\left|\sum_{i} \overrightarrow{\mathrm{r}}_{i}\right| E\right\rangle=\left\langle E_{g}\left|\sum_{i} \overrightarrow{\mathrm{p}}_{i}\right| E\right\rangle,
$$

where $\left|E_{g}\right\rangle$ is the ground-state HS Slater determinant and $-\dot{E}_{g}$ is the binding energy of the photoionized shell. This equality holds for the exact wavefunctions as well, but not for Hartree-Fock wavefunctions since they are derived from a nonlocal potential. $^{3,8}$ We now show that this relation does 
not hold also for our "improved" length and velocity matrix elements, obtained in terms of the HS dipole-matrix elements by multiplying both sides of (1) by $\left\langle E_{g}\right| \sum_{i} \overrightarrow{\mathrm{r}}_{i}$ or $\left\langle E_{g}\right| \sum_{i} \overrightarrow{\mathrm{p}}_{i}$, respectively. Consider the resulting formula for $\left\langle E_{g}\left|\sum_{i} \overrightarrow{\mathrm{p}}_{i}\right| \Psi_{E}\right\rangle$; use (3) to replace HS dipole-velocity by HS dipolelength matrix elements. One would obtain, thereby,

$$
-i\left(E-E_{g}\right)\left\langle E_{g}\left|\sum_{i} \vec{r}_{i}\right| \Psi_{E}\right\rangle,
$$

were it not for an energy-dependent factor $\left(\epsilon-E_{g}\right) /$ $\left(E-E_{g}\right)$ in the integrand. Hence, when improving only excited-state wavefunctions as we do here, we expect length and velocity results for the cross section to diverge. ${ }^{13}$

\section{NOTES ON NUMERICAL PROCEDURE}

(i) As is well known, the expectation value of $r^{-1}$ in a continuum state diverges logarithmically. For this reason, when calculating matrix elements of $V$, terms with asymptotic Coulomb tails $\left(\mathrm{F}^{0}\right.$ Slater integrals, $V_{\mathrm{HS}}$, and $\left.-Z / r\right)$ must be combined to cancel each other.

(ii) The integrals in (1) and (2) imply sums over discrete states, which were accomplished as follows: Levels were summed until they became close spaced, which occurs rapidly. The succeed-

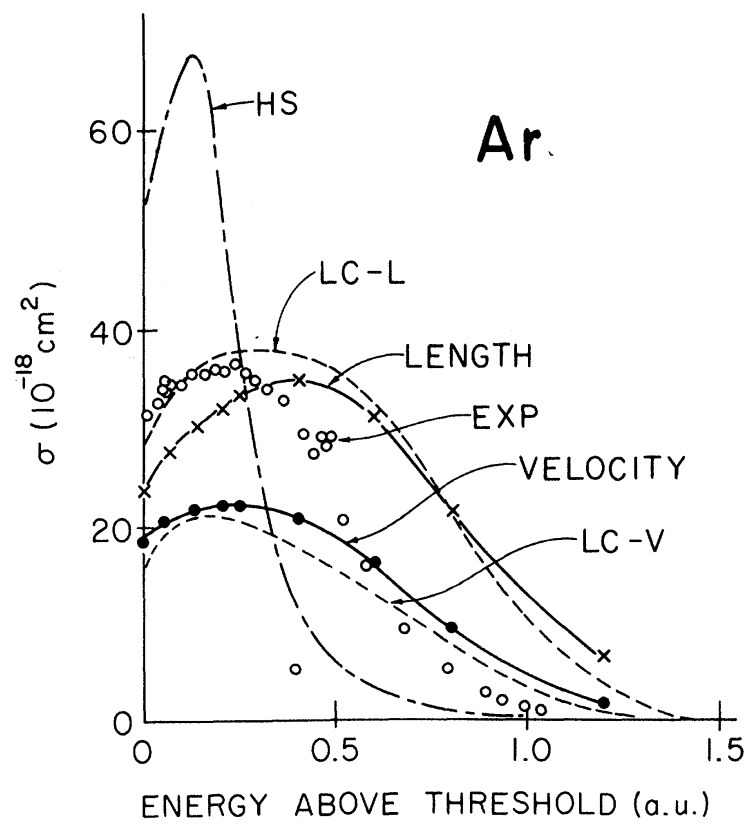

FIG. 1. Photoionization cross sections for the $3 p$ shell in argon. Solid lines: meant to indicate the trend of the present results, which are the crosses (LENGTH) and dots (VELOCITY). LC-L and LC-V: Length and velocity results of L. Lipsky and J. W. Cooper (Ref. 10). HS: Herman-Skillman cross section. EXPT: experimental results of J. A. R. Samson (Ref. 15).

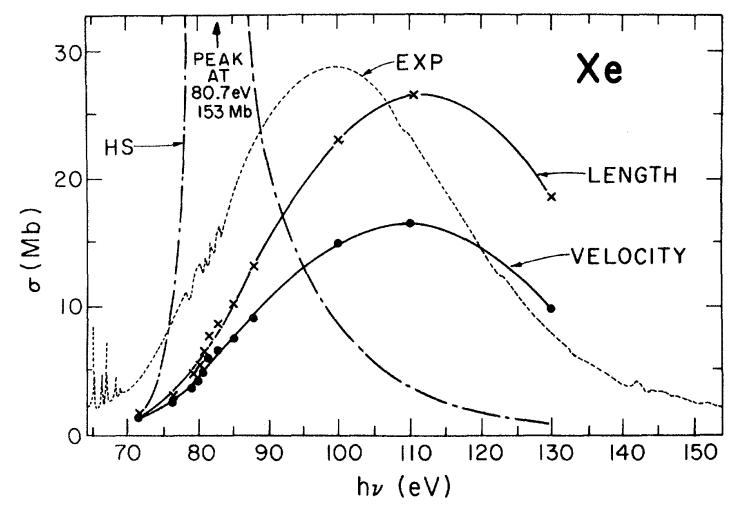

FIG. 2. Photoionization cross sections for the $4 d$ shell in xenon. Solid lines: meant to indicate the trend of the present resulis, which are the crosses (LENGTH) and dots (VELOCITY). HS: Herman-Skillman cross section. EXPT: experimental results of R. Haensel, G. Keitel, P. Schreiber, and C. Kunz (Ref. 16).

ing level (of principal quantum number $n$ and energy $E_{n}$ ) was multiplied by the factor

$$
\left(\frac{d n}{d E}\right)_{E=E_{n}}^{1 / 2}=-\left(2 E_{n}\right)^{-3 / 4},
$$

which gives this discrete level a continuum normalization. ${ }^{14}$ The integration was then started at this discrete energy rather than at threshold.

(iii) For each improved final state $\left|\Psi_{E}\right\rangle$, we must calculate the column of a reaction matrix $K(E)$. This column is obtained by reduction of the set of integral (2) - one for each row index $\epsilon-$ to a set of linear equations in the elements of the column matrix. ${ }^{9}$ Hence, every point we obtain for the cross section involves a separate calculation, and the scatter of points from a smooth curve is a measure of numerical consistency.

\section{RESULTS AND CONCLUSIONS}

We see from Figs. 1 and 2 that our calculations have succeeded in both flattening and broadening the HS cross sections. As expected, length and velocity results disagree by a large factor. (They appear equal at threshold in xenon, but this is because $\sigma_{4 d \rightarrow \epsilon f} \ll \sigma_{4 d \rightarrow \epsilon p}^{\mathrm{HS}}$ at this energy due to the angular momentum barrier. ${ }^{4}$ ) The length cross sections in both cases peak at about the experimental values, but they fail to drop as fast as the experimental cross sections. ${ }^{15,16}$ In particular, the knee in the argon cross section is not reproduced. The velocity curves peak too low, but they overshoot only slightly. For argon, our results compare well with the Hartree-Fock results of Amusia et $a l .,{ }^{2,3}$ and with the calculations of Lipsky and Cooper, ${ }^{10}$ in which interchannel interactions among three channels $\left(3 s^{2} 3 p^{5} \epsilon d, 3 s^{2} 3 p^{5} \epsilon s\right.$, and $\left.3 s 3 p^{6} \epsilon p\right)$ were accounted for in addition to intrachannel in- 
teraction (see Fig. 1).

We conclude that intrachannel interaction in the continuum gives significant improvement in calculated photoionization cross sections near threshold. Interchannel interaction so far seems to have a minor influence on the gross spectral shape of the cross section. Possibly, the sizable remaining discrepancies between experiment and length and velocity results for the cross section can be accounted for by ground-state correlations, by interchannel interactions with series of doubly excited levels, and by the development of consistent formulas for the transition matrix element in approximate calculations.

\section{ACKNOWLEDGMENTS}

I would like to thank Professor U. Fano for numerous valuable discussions and suggestions. I am also indebted to Professor P. L. Altick for very helpful suggestions and for providing me with two of his computer programs.

\footnotetext{
*Work supported in part by U. S. Atomic Energy Commission under Contract No. COO-1674-28.

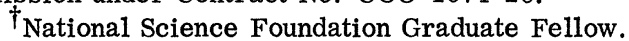

${ }^{1}$ U. Fano and J. W. Cooper, Rev. Mod. Phys. 40, 441 (1968), Sec. 6.

${ }^{2}$ M. Ya. Amusia, N. A. Cherepkov, L. V. Chernysheva, and S. I. Sheftel, Zh. Eksperim. i Teor. Fiz. $\underline{56}, 1897$ (1969) [Soviet Phys. JETP (to be published)].

${ }^{3}$ M. Ya. Amusia, N. A. Cherepkov, L. V. Chernysheva, and S. I. Sheftel, Phys. Letters 28A, 726 (1969).

${ }^{4}$ J. W. Cooper, Phys. Rev. Letters 13, 762 (1964).

${ }^{5}$ F. Herman and S. Skillman, Atomic Structure Calculations (Prentice-Hall, Englewood Cliffs, N. J., 1963).

${ }^{6}$ Reference 1, Sec. 5.3.

${ }^{7}$ J. Goldstone, Proc. Phys. Soc. (London) A239, 267 (1957).

${ }^{8}$ E. S. Chang and M. R. C. McDowell, Phys. Rev. 176 , 126 (1968).

${ }^{9}$ P. L. Altick and E. N. Moore, Phys. Rev. 147, 59
}

\author{
(1966). \\ ${ }^{10}$ L. Lipsky and J. W. Cooper (unpublished); method \\ discussed in Ref. 1. \\ ${ }^{11}$ M. Ya. Amusia, N. A. Cherepkov, and S. I. Sheftel, \\ Phys. Letters 24A, 541 (1967). \\ ${ }^{12}$ W. Brandt, L. Eder, and S. Lundqvist, J. Quant. \\ Spectry. Radiative Transfer 7 , 185 (1967). \\ ${ }^{13}$ Result quoted as St(69) by U. Fano and J. W. Cooper, \\ Rev. Mod. Phys. 41, 724 (1969); this is a generaliza- \\ tion of a similar, unpublished result (for first-order cor- \\ rected HS length and velocity matrix elements) by $\mathrm{S}$. T. \\ Manson. \\ ${ }^{14}$ Reference 1, Sec. 2.4. \\ ${ }^{15}$ J. A. R. Samson, Advan. At. Mol. Phys. 2, 178 \\ (1966). \\ ${ }^{16}$ R. Haensel, G. Keitel, P. Schreiber, and C. Kunz, \\ Deutsches Elektronen-Synchrotron Report No. DESY \\ 69/23, 1969 (unpublished).
}

\title{
Two-State Stueckelberg-Landau-Zener Theory Applied to Oscillatory Inelastic Total Cross Sections*
}

\author{
R. E. Olson \\ Stanford Research Institute, Menlo Park, California 94025 \\ (Received 6 January 1970)
}

\begin{abstract}
The two-state Stueckelberg-Landau-Zener theory of curve crossings can be utilized to explain qualitatively many of the observed asymmetric-inelastic total cross sections. It predicts not only a broad maximum in the inelastic total cross sections as a function of velocity, but also a rapid rise to this maximum followed by a slow decline past the maximum. If the difference in the potentials between the two states possesses an extremum, oscillations superimposed upon the general velocity dependence of the inelastic total cross sections may also be expected because of the existence of a stationary phase. At high velocities, the spacing of these oscillations will be proportional to $v^{-1}$.
\end{abstract}

\section{INTRODUCTION}

The recent measurements of total charge-transfer cross sections by Perel et al..$^{1-3}$ on the alkalialkali systems in the $0.5-10 \times 10^{2}-\mathrm{cm} / \mathrm{sec}$ velocity range have shown prominent regularly spaced oscillations superimposed upon a smooth energy dependence of the cross sections. These oscillations occur in the asymmetric systems 